\title{
PERIODIC FIBONACCI ALGEBRAS
}

\author{
by D. L. JOHNSON and A. C. KIM
}

(Received 25th April 1990)

\begin{abstract}
Fibonacci algebras are groups equipped with an extra unary operation $\phi$ that satisfies a Fibonacci-type law. We described in an earlier paper the free objects in the resulting varieties, and here we do the same in the case when $\phi$ is assumed to be periodic. They turn out to be central extensions of Burnside groups with finite kernels whose orders can be expressed in terms of the resultants of certain polynomials.
\end{abstract}

1980 Mathematics subject classification (1985 Revision): 08B20, 20 F50.

\section{Introduction}

We are concerned with the variety $\mathfrak{B}(m, n)$ of universal algebras $G$ of the following type: $G$ is a group equipped with a unary operation $\phi$ that is an automorphism of $G$ and satisfies the one-variable laws

$$
\begin{gathered}
x x \phi \ldots x \phi^{m-1}=x \phi^{m}, \\
x \phi^{n}=x
\end{gathered}
$$

where $m$ and $n$ are positive integers and, as in the Polish notation, any occurrence of $\phi$ or one of its powers is understood to apply only to the symbol immediately preceding it. It seems natural to call such objects Fibonacci algebras, or $\phi$-algebras for short, and the $\mathfrak{B}(m, n)$ Fibonacci varieties. Our main object of study is the free object $V_{d}(m, n)$ of rank $d$ in $\mathfrak{B}(m, n)$. (We assume $m>1$ to avoid triviality.)

\section{The problem}

The case when $m=2$ is covered comprehensively in [3], where it is shown that the monogenic free algebra $V_{1}(2, n)$ is isomorphic to the abelianised Fibonacci group $A(2, n)$ studied in [1] and defined as the derived factor group of the Fibonacci group

$$
F(2, n)=\left\langle x_{1}, x_{2}, \ldots, x_{n} \mid x_{1} x_{2}=x_{3}, \ldots, x_{n-1} x_{n}=x_{1}, x_{n} x_{1}=x_{2}\right\rangle .
$$

The $A(2, n)$ are all finite and their invariant factors are given explicitly in [3] in terms of the Fibonacci numbers. 
The case of arbitrary $m$ is treated in [4], where it is shown (Theorem 3) that the elements $x^{-1} x \phi, x \in G$, form a central subgroup, called $G \theta$ here as in [2], and that the quotient group $G / Z$ lies in the Burnside variety $\mathfrak{B}(m-1)$ of groups of exponent dividing $m-1$. There is thus a central extension

$$
0 \rightarrow G \theta \rightarrow G \rightarrow G^{\phi} \rightarrow 1
$$

where $\theta: G \rightarrow G$ is given by $x \theta=x^{-1} x \phi$, and $G^{\phi}$ is the biggest $\phi$-trivial factor-algebra of $G$. In particular, there is a central extension

$$
0 \rightarrow Z_{d}(m, n) \rightarrow V_{d}(m, n) \stackrel{v}{\rightarrow} B_{d}(m-1) \rightarrow 1
$$

where $B_{d}(m-1)$ is the free object of rank $d$ in $\mathfrak{B}(m-1)$. It is stated in [4] that $Z_{d}(m, n)$ is free abelian of rank $d_{m}$ when $n=0$ and finite when $n>0$. The first of these assertions is proved in [2, Corollary 4.4], and the second is proved below.

\section{First reduction}

We regard $V_{d}(m, n)$ as the $d$ th free power (in $\mathfrak{B}(m, n)$ ) of $V_{1}(m, n)$ and invoke the results of [2] to reduce our problem to the monogenic case.

Corollary 4.2 of [2] asserts that the central extension (4) is well-behaved under the formation of free products: for $G, H \in \mathfrak{B}(m, 0)$, there is a central extension

$$
0 \rightarrow G \theta \times H \theta \rightarrow G *_{\phi} H \rightarrow G^{\phi}{ }{ }_{B} H^{\phi} \rightarrow 1,
$$

where $\times, *_{\phi}$ and $*_{B}$ denote products in the varieties $\mathfrak{U}$ (of abelian groups), $\mathfrak{B}(m, 0)$ and $\mathfrak{B}(m-1)$, respectively.

Now the imposition of (2) has no effect on the right-hand term, and factoring out by the relations it induces commutes with the formation of direct products. (6) can be regarded as a central extension in $\mathfrak{B}(m, n), n>0$, with $*_{\phi}$ suitably re-interpreted. A simple induction on $d$ now gives the following results.

Lemma 1. For all $m, n, d \in \mathbb{N}$, the kernel $Z_{d}(m, n)$ is the $d$ th direct power of $Z_{1}(m, n)$.

\section{Second reduction}

The extension (5) is described explicitly in $\S 3$ of [2] in the case when $d=1$ and $n=0$ : $V_{1}(m, 0)$ is the result of imposing the law (1) on the free abelian group with basis $\left\{x_{i} \mid i \in \mathbb{Z}\right\}$, where $x_{i} \phi=x_{i+1}$ for all $i$. But here, we impose the law (2) first, giving the free abelian group on $X=\left\{x_{1}, \ldots, x_{n}\right\}$, and the subsequent imposition of (1) yields the group with presentation

$$
\langle X \mid R, C\rangle
$$


where

$$
\begin{gathered}
C=\left\{x_{i} x_{j}=x_{j} x_{i} \mid 1 \leqq i<j \leqq n\right\}, \\
R=\left\{x_{i} x_{i+1} \ldots x_{i+m-1}=x_{i+m} \mid 1 \leqq i \leqq n\right\},
\end{gathered}
$$

with subscripts reduced modulo $n$. Noting that $\langle X \mid R\rangle$ is just the Fibonacci group $F(m, n)$, we have proved the following result, which is Theorem 1 of [4].

Lemma 2. The monogenic free $\phi$-algebra $V_{1}(m, n)$ is isomorphic to the abelianized Fibonacci group $A(m, n)$.

\section{The solution}

It is shown in [1] that $A(m, n)$ is the $n$-generator group whose relation matrix is the circulant matrix determined by the polynomial

$$
f(t)=1+t+\cdots+t^{m-1}-t^{m}
$$

so that $A(m, n)$ has order

$$
\prod_{k=0}^{n-1}\left|f\left(\omega^{k}\right)\right|, \quad \omega=e^{2 \pi i / n}
$$

which is just the resultant res $(f, g)$ of the polynomials $f(t)$ and $g(t)=1-t^{n}$. Because $f$ and $g$ have no common root, this is a positive integer (so that $A(m, n)$ is a finite group) which is divisible by $m-1=f(1)$. As the kernel $Z_{1}(m, n)$ has index $m-1$ in $V_{1}(m, n)$, this may be combined with Lemmas 1 and 2 to give our main result.

Theorem. For all $d, m, n \in \mathbb{N}$ with $m>1$, the kernel $Z_{d}(m, n)$ of the natural map

$$
v: V_{d}(m, n) \rightarrow B_{d}(m-1)
$$

is a finite abelian group of order $r_{m, n}^{d}$, where $r_{m, n}$ is the resultant of the polynomials

$$
1+t+\cdots+t^{m-1}-t^{m} \text { and } 1+t+\cdots+t^{n-1} .
$$

The values of $r_{m, n}$ are given explicitly in [1] for certain favourable values of $m$ and $n$.

Acknowledgements. Both authors are grateful to the Royal Society of London, the Korean Science and Engineering Foundation, and the British Council, without whose continued support this collaboration would not have been possible. 


\section{REFERENCES}

1. D. L. Johnson, A note on the Fibonacci groups, Israel J. Math. 17 (1974), 277-282.

2. D. L. Johnson and ANn Chi Kim, Free Fibonacci Algebras, Bull. Austral. Math. Soc. 40, (1989), 235-241.

3. AnN Сhi Kiм, Fibonacci variaties, Bull. Austral. Math. Soc. 19 (1978), 191-196.

4. Ann Chi Kim, B. H. Neumann and A. H. Rhemtulla, More Fibonacci varieties, Bull. Austral. Math. Soc. 22 (1980), 385-395.

Mathematics Department

UNIVERSITY OF NOTTINGHAM

NotTingham NG7 2RD

ENGLAND

\author{
Mathematics Department \\ Busan National University \\ BUSAN 607 \\ KOREA
}

